\title{
Environmental Management
}

\section{Estimating the Mass of Chemicals Associated with Ocean Plastic Pollution to Inform Mitigation Efforts}

\author{
Hannah L De Frond, $+\neq$ Erik van Sebille, $\neq$ J Mark Parnis, $\$$ Miriam L Diamond,// Nicholas Mallos, \# \\ Tony Kingsbury, t广 and Chelsea M Rochman*tt \\ †Environment Department, University of York, York, United Kingdom \\ Institute for Marine and Atmospheric research, Utrecht University, Utrecht, Netherlands \\ $\S$ Chemical Properties Research Group, Department of Chemistry, Trent University, Peterborough, Ontario, Canada \\ //Department of Earth Sciences, University of Toronto, Toronto, Ontario, Canada \\ \#Ocean Conservancy, Portland, Oregon, USA \\ †TKingsbury Ilc Consulting, Auburn, California, USA \\ $\$$ Department of Ecology and Evolutionary Biology, University of Toronto, Toronto, Ontario, Canada
}

\begin{abstract}
Plastic pollution in the marine environment is well documented. What remains less recognized and understood are the chemicals associated with it. Plastics enter the ocean with unreacted monomers, oligomers, and additives, which can leach over time. Moreover, plastics sorb organic and inorganic chemicals from surrounding seawater, for example, polychlorinated biphenyls (PCBs) and metals. Thus, interception and cleanup of plastics reduces the amount of chemical contaminants entering or reentering the oceans and removes those already present. Here, we estimate 1) the mass of selected chemical additives entering the global oceans with common plastic debris items, and 2) the mass of sorbed chemicals (using PCBs as a case study) associated with microplastics in selected locations. We estimate the mass of additives that entered the oceans in 2015 as constituents of 7 common plastic debris items (bottles, bottle caps, expanded polystyrene (EPS) containers, cutlery, grocery bags, food wrappers, and straws or stirrers). We calculate that approximately 190 tonnes ( $t$ ) of 20 chemical additives entered the oceans with these items in 2015. We also estimate the mass of PCBs associated with microplastics in 2 coastal (Hong Kong and Hawaii) and 2 open ocean (North Pacific and South Atlantic gyres) locations, as comparative case studies. We find that the mass of chemicals is related to the mass of plastics in a location, with greater mass of PCBs closer to the source (i.e., land), where there is more plastic per unit area compared to the open ocean. We estimate approximately 85000 times more PCBs associated with plastics in an average 4.5-km stretch of beach in Hong Kong than from the same size transect in the North Pacific gyre. In conclusion, continuing efforts for plastic interception and cleanup on shorelines effectively reduces the amount of plastic-related chemicals entering and/or reentering the marine environment. Integr Environ Assess Manag 2019;15:596-606. () 2019 SETAC
\end{abstract}

Keywords: Additives Leaching Microplastic PCBs Plastic pollution

\section{INTRODUCTION}

Plastic contamination of the marine environment represents a significant and growing global problem (Derraik 2002; Gregory and Andrady 2003; Jambeck et al. 2015). Worldwide annual plastic production increased from 2 million tonnes (MT) in 1950 to 380 MT in 2015 (Geyer et al. 2017). Of all plastics produced to date, $79 \%$ are estimated to be in landfills or present as debris in the natural environment (Geyer et al. 2017). Jambeck et al. (2015) estimate that of the $275 \mathrm{MT}$ of plastic waste generated in 2010 alone, 4.8 to $12.7 \mathrm{MT}$ entered the ocean, becoming plastic pollution. If the present trends in production, use,

This article contains online-only Supplemental Data.

* Address correspondence to chelsea.rochman@utoronto.ca

Published 22 March 2019 on wileyonlinelibrary.com/journal/ieam. and waste management of plastics continue, it is predicted that up to $28 \mathrm{MT}$ of plastic will enter the oceans annually by 2025 (Jambeck et al. 2015).

The accumulation of plastic in the ocean is more than just an eyesore. Plastic debris threatens the health and survival of marine life via ingestion, entanglement, smothering, and the transport of nonnative species (Derraik 2002; Gregory 2009). Such physical hazards have been observed and researched for decades; however, researchers have only recently begun investigating chemical hazards. Plastic marine debris acts as a sink for many hazardous organic chemicals and metals found in seawater (Ogata et al. 2009; Engler 2012; Holmes et al. 2012), and therefore may act as a vector for long-range chemical transport. Included in the chemicals that sorb from marine waters to plastic marine debris are persistent organic pollutants (POPs; e.g., polychlorinated biphenyls [PCBs], polycyclic aromatic hydrocarbons [PAHs], polybrominated 
diphenyl ethers [PBDEs], hexachlorocyclododecane [HBCD]) and other substances that are listed as priority pollutants in many countries (e.g., Hg, Pb, and nonylphenols) (Ogata et al. 2009; Hirai et al. 2011; Holmes et al. 2012). Several of these chemicals are no longer in production but persist in the environment today. For example, PCBs were last produced in the late 1970s and early 1980s in Western countries and in 1990 in Russia (Breivik et al. 2007). Still, because PCBs are extremely persistent, they are found in virtually all locations and parts of the global ecosystem (Jepson and Law 2016) and are found on nearly all microplastic pollution recovered from oceans and coastlines (Rios et al. 2007; Hirai et al. 2011).

Recently, sorbed chemicals have dominated discussions about the toxicological risk of marine plastic pollution. What may be more relevant with respect to plastics are the chemicals added during manufacturing, which may be subsequently released into the ocean via leaching or weathering (Engler 2012; Gewert et al. 2015; Suhrhoff and Scholz-Böttcher 2016). Plastic polymers themselves are generally considered biochemically inert materials. However, a variety of chemicals are combined during the manufacture of plastics to provide specific functionalities, such as to enhance performance and improve the properties of the resulting products (Geyer et al. 2017). Chemical additives include plasticizers, colorants, UV stabilizers, antioxidants, and antimicrobials (Thompson et al. 2009; Hammer et al. 2012; Geyer et al. 2017). Flame retardants can also be added to comply with flammability standards. Some of these chemicals are nonreactive additives. Others are reactive, but some can remain unreacted in the final plastic polymer. Both are able to leach from the plastic (Teuten et al. 2009). Moreover, monomeric feedstock materials and partially polymerized oligomers of the polymer are often present and able to leach, due to incomplete polymerization (Lithner et al. 2011). The fate of the plastic additives may therefore differ from the fate of the plastic itself. For example, these released chemicals may bioconcentrate in organisms, although the plastic itself might drift away. This is a matter of concern because some of the chemicals in plastics have been discussed in the literature as a potential hazard when they leach (Westerhoff et al. 2008; Wik and Dave 2009; Rani et al. 2014; Tawfik and BaAbdullah 2014), and some are listed as hazardous by the United Nations Globally Harmonized System of Classification and Labelling of Chemicals (Lithner et al. 2011; UNECE 2013).

Estimates suggest nonfiber plastics contain on average $7 \%$ additives by mass (Geyer et al. 2017). Thus, if 28 MT of plastic waste is predicted to enter the oceans in 2050 (Jambeck et al. 2015), about 2 MT of chemical additives will be carried with it. Since the middle of the 20th century, the production and use of additives in plastics has increased exponentially (Binetti et al. 2008); therefore this number is only expected to increase with the continued production of plastics and resulting masses of mismanaged waste. If current rates of plastic production continue, a total of 2000
MT of additives will have been produced by the end of 2050 (Geyer et al. 2017).

When plastics become marine debris, they act as both a source of additives and a sink over time for other chemical contaminants (Teuten et al. 2009). As such, prevention and removal of marine plastic pollution are also a means for the prevention and removal of chemical contaminants from our oceans. Therefore, while we continue to investigate the fate and hazards of chemicals associated with plastics, it is also important to think about how this may inform solutions and management.

Our main objective was to estimate the mass of additives and sorbed chemicals that can be prevented from entering or removed from the oceans along with plastic pollution via cleanup efforts. Because such efforts are difficult to do globally, and because plastic debris is already ubiquitous in the marine environment, our second goal was to identify a strategy for cleanup efforts. We used case studies to estimate amounts of plastics and associated chemicals that could be removed or prevented from reaching the oceans under specific scenarios. First, we calculated the mass of chemical additives entering the ocean each year embodied within 7 common plastic debris items. Next, we estimated the mass of sorbed chemicals associated with microplastics within the North Pacific Gyre, South Atlantic Gyre, and on the coastlines of Hong Kong and Hawaii, using PCBs as an example. Below, we share our results and discuss how they may inform management strategies related to removal of plastic debris from shorelines versus the open ocean.

\section{METHODS}

Quantification of additives associated with plastics entering the oceans

Total mass of 7 plastic items entering the ocean each year. As a case study, we estimated the total mass of chemical additives that enters the ocean each year within 7 common plastic marine debris items. The items studiedbeverage bottles, bottle caps, expanded polystyrene (EPS) food and drink containers, cutlery, grocery bags, straws or stirrers, and food wrappers-are all among the top 10 debris items most commonly found and collected during beach cleans (Ocean Conservancy 2017). The remaining items on this list (glass bottles, other plastic bags, and plastic lids) were not included because information on additives was unavailable, or in the case of glass bottles, the item was not made of plastic and therefore was not applicable to the present study.

To calculate the total mass of additive carried by the plastic, we first estimated the total mass of each of these plastic products that enters the ocean each year, $M_{T i}$ (tonnes/year [t/y]) (Figure 1). Because of a lack of data, we estimated $\mathrm{M}_{\mathrm{Ti}}$ from the Ocean Conservancy's (2014) International Coastal Clean-up (ICC) data, which includes the total number and average mass of each of these products and many others collected at $>6000$ beach cleanups 


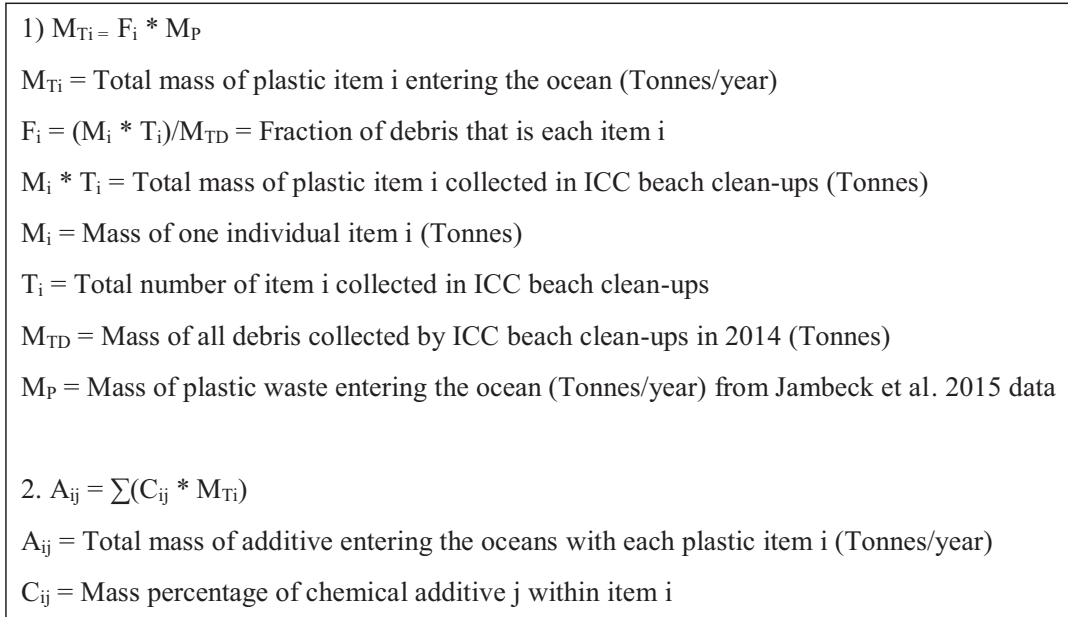

Figure 1. Equations used to calculate the total mass $(t)$ of each plastic debris item that enters the ocean per year $\mathrm{M}_{\mathrm{Ti}}(\mathrm{t} / \mathrm{y})$, repeated with data from Jambeck et al. (2015) for 2015, 2020, 2025 under scenarios of $15 \%, 25 \%$, and $40 \%$ of mismanaged plastic waste entering the ocean (1). Equation used to determine $A_{i j}$; the total mass of each chemical additive $(t / y)$ entering the oceans with each plastic debris item (2). worldwide. We used beach litter data, making the assumption that the litter on shorelines (which includes beaches and river banks) is indicative of the litter than goes into the ocean each year. This litter includes a mix of durable and single-use goods, which is likely representative of the litter that goes into the ocean from several sources. For our calculations, we used ICC data from the year 2014 because this was the most comprehensive and recent data available to use for estimations of marine plastic and related additive loads in 2015. We assessed the representativeness of 2014 data by comparing data from 2012 through 2017 and found that the total number of items collected in 2014 were within 1 standard deviation (+/-) of the mean for the years 2012 to 2017. The ICC data state the number of items collected via cleanup, but not the total mass collected for each item. Thus, to estimate the total mass of each of these plastic products that enter the oceans each year, $M_{T i}$, we first multiplied the average weight of each product, $M_{i}(t)$ (Supplemental Data Appendix S1), by the total number of this product that was collected during beach cleans (excluding fragments), $T_{i}$, to calculate the total mass collected in 2014 for each item globally. We then divided this by the total mass of all items collected by ICC in 2014, $\mathrm{M}_{\text {TD }}$ (excluding the weight of all nonplastic items $\approx 5.33$ million kg; see Supplemental Data Appendix S2), to calculate the fractional mass of each product collected along coastlines globally, F.

Next, from the total amount of plastic waste in 2010, Jambeck et al. (2015; Supplemental Data Appendix S3) estimated the amount of mismanaged waste that became marine debris under 3 scenarios: low (15\% entering the ocean), medium (25\%), and high (40\%). The data set used also included projections for the years 2015, 2020, and 2025. Due to the lack of available data, we assumed that the proportions of different types of plastic products found as beach litter (ICC) are representative of the proportions of plastic products found in the mismanaged waste that becomes marine debris. For each item, we multiplied $\mathrm{F}$ by the total mass of plastic estimated to become marine debris in that year, $M_{P}(t / y)$. This final step was repeated to provide an estimated total mass of each plastic item entering the ocean each year for 2015, 2020, and 2025 under the 15\%, $25 \%$, and $40 \%$ scenarios.

Items such as cutlery, bottles, and food wrappers can be made from different types of plastics containing different chemical additives. To the best of our knowledge, information on these proportions is not publicly available. Therefore, for these 3 debris items we estimated the proportion of the total amount of litter that constitutes each type of plastic based on personal communications with people in industry (see Acknowledgment) and assumed their information to be representative. The total mass of the item entering the ocean each year $\left(\mathrm{M}_{\mathrm{Ti}_{\mathrm{i}}}\right)$ was divided, giving 2 proportional masses for the 2 different types of plastic that are used most commonly for that item. For plastic cutlery, we estimated that $80 \%$ is polypropylene (PP) and $20 \%$ is general-purpose polystyrene (GPPS). For plastic bottles, we estimated that $80 \%$ are polyethylene terephthalate (PET) and $20 \%$ are highdensity polyethylene (HDPE). For food wrappers, we estimated that production is split equally between PET, low-density polyethylene (LDPE), and polypropylene (PP). We also assumed all grocery bags found in beach cleans were HDPE and that all bottle caps were PP.

Mass of additive entering the ocean each year with each plastic item. Data on the mass percentage of additives commonly used within the selected plastic items were provided by leading plastic producers and members of the American Chemistry Council (ACC) Plastics Division. In addition, the mass percentages of additives for some items were sourced from the peer-reviewed literature (See Supplemental Data Appendices S4 and S5 for a list of additives, CAS numbers and weight percentages in each item). Below, we present chemical additive estimates for each of the 7 items based upon the amount of each item entering the oceans in $2015\left(\mathrm{M}_{\mathrm{T}_{\mathrm{i}}}\right)$ under the medium (25\% mismanaged waste) scenario (see Supplemental Data Appendices S6-S13 for alternative scenarios). We multiplied 
the mass percentage of chemical $j$ in each plastic item $i, C_{i j,}$ by the total mass of that item entering the ocean per year $\left(M_{T_{i}}\right)$, to provide the final value for each additive, $A_{i j}(t / y)$, the mass of each chemical additive that is estimated to have entered the oceans in $\mathbf{2 0 1 5}$ with each plastic debris item, $i$ (Figure 1).

Only 7 debris items were included within the scope of the present study. Most are food grade and thus have fewer additives than other types of plastics. Further, the list of chemicals we obtained for the present study was not inclusive of all additives used in these items. Additives may also be added by plastic converters, that is, those that produce products from raw material pellets. Other additives may include colorants, release agents, inks, and dyes. We have not included these additives in the present study. As such, we have underestimated the mass and variety of additives associated with plastic debris globally. In addition, we note that our results may have overestimated some specific chemicals, for example, mold-release agents, given that some chemicals may be retained as a residue on the mold, whereas some will remain within the plastic itself.

\section{Quantification of PCBs on microplastics in the ocean}

Quantification of microplastics in 4 locations. As a case study for adsorption of (nonadditive) POPs in ocean waters, we chose to focus on PCBs as a representative chemical family. Here, we are using only microplastics, instead of using larger plastic products because data for chemical sorption tends to be available only for microplastics. To quantify the reservoir of PCBs associated with marine microplastics, we chose 2 coastal locations as case studies, Hawaii and Hong Kong, and 2 open ocean locations, the North (N) Pacific and South (S) Atlantic subtropical gyres. These locations were selected due to the availability of data for both microplastic pollution and PCBs. Hawaii receives large quantities of plastic debris on its coastlines, carried by currents from the North Pacific Subtropical Convergence Zone (Carson et al. 2013). Hong Kong is a special administrative region (SAR) of China, and China is the top producer of plastic waste globally (Jambeck et al. 2015). The $\mathrm{N}$ Pacific gyre is well known to have the largest quantities of open ocean plastic (Eriksen et al. 2013), and the S Atlantic gyre is known to have less open ocean plastic than other oceanic gyres (Eriksen et al. 2013). These locations are known to collect large amounts of plastic debris, and therefore they are areas where substantial amounts of microplastics could be removed.

Ranges of microplastic pollution on coastlines $\left(\mathrm{g} / \mathrm{m}^{2}\right)$ were sourced from Carson et al. (2011) for Hawaii and from Fok and Cheung (2015) for Hong Kong. For the gyres, the 95\% confidence minimum and maximum masses of microplastics in the area were estimated using the model developed by van Sebille et al. (2015). These numbers were used to estimate the amount of microplastics that could be removed from the oceans or beaches within equal-sized transects. As a starting reference, we used Kamilo Beach, Hawaii, which has a coastline of $4500 \mathrm{~m}$ in length. To determine the mass of all the microplastics there, we started with the minimum and maximum mass of microplastics within a $5-\mathrm{cm}^{3}$ sample of sand (Carson et al. 2011). Assuming this is consistent across the beach, we extrapolated to estimate the minimum and maximum amount of microplastics within a $4500 \mathrm{~m} \times 1$ $\mathrm{m}$ transect. We then repeated these calculations for the 3 other locations by using the minimum and maximum amounts of microplastics $\left(\mathrm{g} / \mathrm{m}^{2}\right)$ found in each region to calculate the range of microplastics found within a $4500 \mathrm{~m} \times$ $1 \mathrm{~m}$ transect.

Quantification of PCBs within microplastics. Ranges of PCB concentrations in microplastics (ng/g) were obtained from the International Pellet Watch (IPW) for Hawaii and Hong Kong (IPW 2017), from Hirai et al. (2011) for the N Pacific gyre, and from Rochman et al. (2014) for the $S$ Atlantic gyre. To determine the range of the mass of PCBs associated with the microplastics in each transect, we multiplied the minimum amount of microplastics (g) by the lowest concentrations of PCBs (ng/g) found on microplastics in each region. We then repeated this for the maximum amount of microplastics and maximum PCB concentrations. The data included the sum of congeners PCB \#66, 101, 105, $110,118,128,138,149,153,170,180,187$, and 206. This provided a range for the total mass of PCBs (ng) collected if all microplastics were removed within the $4500 \mathrm{~m} \times 1 \mathrm{~m}$ transect at each location. As noted above, our estimates are based on microplastics only and not on macroplastics. This is because the PCB concentrations used are from microplastics $(<5 \mathrm{~mm})$ only, and we did not want to make any assumptions about concentrations of PCBs on larger plastic debris. Therefore, the numbers stated within these results underestimate the total mass of PCBs within plastic debris at each location because we are not considering the large plastic debris that could be removed.

\section{RESULTS AND DISCUSSION}

Prevention of chemical pollutants with prevention of plastic debris

We estimate that the total weight of beverage bottles, bottle caps, EPS food and drink containers, cutlery, grocery bags, straws or stirrers, and food wrappers entering the ocean in 2015 was approximately 87000 t (Table 1; 25\% scenario). As such, our calculations account for only $1 \%$ of the approximately $8 \mathrm{MT}$ of plastic that is estimated to enter the ocean each year (Jambeck et al. 2015). The remaining $99 \%$ of plastic debris by weight entering the ocean is likely to comprise larger and heavier nonfood items such as tires and construction materials, which are more likely to contain chemical additives in greater masses. Therefore, our results are likely a considerable underestimation of total load of chemical additives entering the marine environment with plastic debris. 


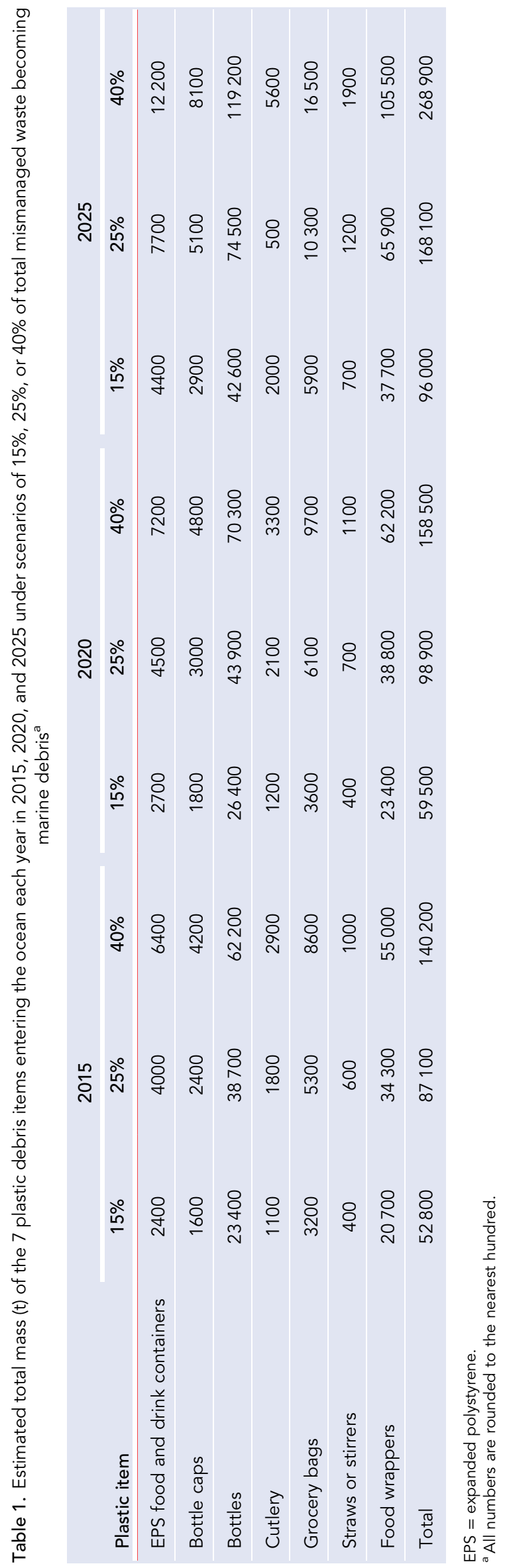


Table 2. Total mass ( $\mathrm{t}$ ) of chemical additive entering the marine environment in 2015 with 7 items of plastic debris under a medium scenario (25\%) of mismanaged plastic waste entering the oceans

\begin{tabular}{|c|c|c|c|c|c|c|c|c|c|c|c|}
\hline \multirow[b]{2}{*}{ Additive $^{a}$} & \multicolumn{11}{|c|}{ Plastic debris item } \\
\hline & $\begin{array}{c}\text { EPS } \\
\text { containers }\end{array}$ & $\begin{array}{l}\text { Bottle } \\
\text { caps }\end{array}$ & $\begin{array}{l}\text { PET } \\
\text { bottles }\end{array}$ & $\begin{array}{l}\text { HDPE } \\
\text { bottles }\end{array}$ & $\begin{array}{l}\text { GPPS } \\
\text { cutlery }\end{array}$ & PP cutlery & $\begin{array}{l}\text { Grocery } \\
\text { Bags }\end{array}$ & $\begin{array}{l}\text { PET food } \\
\text { wrappers }\end{array}$ & $\begin{array}{l}\text { LDPE } \\
\text { food } \\
\text { wrappers }\end{array}$ & $\begin{array}{l}\text { PP food } \\
\text { wrappers }\end{array}$ & $\begin{array}{l}\text { Straws } \\
\text { or } \\
\text { stirrers }\end{array}$ \\
\hline $2082-79-3^{b}$ & 1.6 & 0.05 & - & - & - & 0.72 & 1.3 & - & 5.7 & 0.3 & 0.02 \\
\hline $31570-04-4^{c}$ & 3 & - & 62 & - & - & 0.36 & 1.6 & 22.9 & - & 4 & 0.2 \\
\hline $26741-53-7^{d}$ & - & 0.6 & - & - & - & 0.58 & - & - & - & - & - \\
\hline Sodium benzoate & - & 0.6 & - & - & - & 0.43 & - & - & - & - & - \\
\hline $\begin{array}{l}\text { Glyceryl } \\
\text { monostearate }\end{array}$ & - & 4.7 & - & - & - & 1.8 & - & - & - & - & - \\
\hline $1709-70-2^{e}$ & - & 1.3 & - & - & - & - & - & - & - & - & 0.2 \\
\hline Erucamide & - & 0.7 & - & - & - & - & - & - & - & - & - \\
\hline Antimony trioxide & - & - & 6.2 & - & - & - & - & - & - & - & - \\
\hline $6683-19-8^{f}$ & - & 0.6 & 15.5 & 5.4 & - & 0.14 & 1.6 & 5.7 & - & 11.4 & 0.2 \\
\hline Acetaldehyde & - & - & 0.2 & - & - & - & - & - & - & - & - \\
\hline Zinc stearate & - & - & - & 1.2 & 0.5 & 0.43 & 2.7 & - & - & - & 0.2 \\
\hline Styrene & - & - & - & - & 3.6 & - & - & - & - & - & - \\
\hline White mineral oil & - & - & - & - & 8.7 & - & - & - & - & - & - \\
\hline $\begin{array}{l}\text { Ethylene bis } \\
\text { stearamide }\end{array}$ & - & - & - & - & 0.004 & - & - & - & - & - & - \\
\hline $65447-77-0^{9}$ & - & - & - & - & - & 0.36 & - & - & - & - & - \\
\hline Calcium stearate & - & - & - & - & - & - & 5.4 & - & - & - & - \\
\hline $\begin{array}{l}\text { Aluminum } \\
\text { magnesium } \\
\text { hydroxide } \\
\text { carbonate }\end{array}$ & - & - & - & - & - & - & - & - & - & 2.9 & 0.06 \\
\hline Monoolein & - & - & - & - & - & - & - & - & - & - & 1.3 \\
\hline Talc & - & - & - & - & - & 2.9 & - & - & - & - & - \\
\hline
\end{tabular}

EPS = expanded polystyrene; GPPS = general-purpose polystyrene; HDPE = high-density polyethylene; PET = polyethylene terephthalate; PP = polypropylene.

${ }^{a}$ CAS numbers are used where chemical names are long.

b 2082-79-3: Octadecyl 3-(3,5-di-tert-butyl-4-hydroxyphenyl)propionate.

c 31570-04-4: tris(2,4-ditert-butylphenyl) phosphite.

d 26741-53-7: 3,9-bis(2,4-di-tert-butylphenoxy)-2,4,8,10-tetraoxa-3,9-diphosphaspiro[5.5]undecane.

e 1709-70-2: 1,3,5-trimethyl-2,4,6-tris(3,5-ditert-butyl-4- hydroxybenzyl)benzene.

f 6683-19-8: Pentaerythritol tetrakis(3-(3,5-di-tert-butyl-4-hydroxyphenyl)propionate).

g 65447-77-0: Poly(4-hydroxy-2,2,6,6-tetramethyl-1-piperidineethanol-alt-1,4-butanedioic acid).

The amount of plastic marine debris is predicted to increase in future years under a business-as-usual scenario, as human populations and annual plastic production continue to grow (Jambeck et al. 2015; Supplemental Data Appendix S3). As such, by 2025 the total mass of the 7 items may increase to as much as $268900 \mathrm{t}$ (Table 1; 40\% scenario).

In regard to additives, when combined, these 7 plastic items were estimated to contribute approximately $190 \mathrm{t}$ of chemical additives in 2015 (25\% scenario; see Table 2 for individual additives and amounts), with the potential to leach into the ocean. This number could almost double to 370 t by 2025 (see Supplemental Data Appendices S6-S13 for alternative minimum and maximum scenarios). As a result, simply preventing this material from entering the marine environment ensures the mitigation of at least some chemicals from entering the oceans, in turn mitigating some chemical exposure for marine organisms.

The leaching potential of a chemical additive from a plastic product depends on several chemical and environmental parameters (Teuten et al. 2009; Suhrhoff and ScholzBottcher 2016). Koelmans et al. (2016) suggest that most 
Table 3. Concentration ranges of PCBs within microplastic pellets (ng/g) and the range of microplastics $\left(\mathrm{g} / \mathrm{m}^{2}\right)$ for Hawaii, Hong Kong, North Pacific gyre, and South Atlantic gyre

\begin{tabular}{|lcc|}
\hline Region & PCBs $(\mathrm{ng} / \mathrm{g})^{\mathrm{a}}$ & Microplastics $\left(\mathrm{g} / \mathrm{m}^{2}\right)^{\mathrm{b}}$ \\
\hline Hawaii & $0.1-10$ & Nondetect to $1.57 \times 10^{3}$ \\
\hline Hong Kong & $10-757$ & $8 \times 10^{-4}$ to $2.49 \times 10^{2}$ \\
\hline North Pacific gyre & $1-78$ & $1.7 \times 10^{-6}$ to $5.8 \times 10^{-6}$ \\
\hline South Atlantic gyre & $0.2-1.4$ & $1 \times 10^{-7}$ to $1.5 \times 10^{-6}$ \\
\hline
\end{tabular}

${ }^{a}$ Ranges of PCB concentrations in microplastics (ng/g) sourced from Hawaii and Hong Kong (IPW 2017), North Pacific gyre (Hirai et al. 2011), South Atlantic gyre (Rochman et al. 2014).

${ }^{b}$ Ranges of microplastic pollution on coastlines $\left(\mathrm{g} / \mathrm{m}^{2}\right)$ sourced from Hawaii (Carson et al. 2011), Hong Kong (Fok and Cheung 2015), North Pacific gyre and S Atlantic gyre (van Sebille et al. 2015).

hydrophobic organic compounds, including plastic additives and plasticizers, will reach equilibrium concentrations between ocean water and plastic within $2 \mathrm{y}$. They also estimate that $80 \%$ to $90 \%$ of plastic in the ocean is older than 2 to $4 \mathrm{y}$. As such, we would expect most chemical additives in most plastics to have reached equilibrium with constituent chemicals in the ocean's surface waters. Moreover, many of these plastics undergo weathering and fragmentation, which facilitates faster leaching and attainment of equilibrium (Engler 2012; Gewert et al. 2015; Suhrhoff and Scholz-Böttcher 2016). Therefore, although many plastic additives are intended to remain inside the matrix, it may be assumed that, over time, additives will leach from the plastic into ocean waters. Thus, it is relevant to consider the potential toxicity of additives in addition to the plastic itself, especially because concentrations of plastics, and thus associated additives, are expected to increase.

Many of the additives discussed in the present study have low toxicity to aquatic organisms (see Supplemental Data Appendix S14 for more information on toxicity to freshwater Daphnia magna, a standard toxicity test species). Many other types of marine litter that can be removed from beaches each contain their own unique mixture of chemical additives. Some of that litter originates from commercial products that contain more toxic mixtures of additives than others. For example, concerns have arisen from the leaching of chemicals from the filters of smoked cigarettes (Wright et al. 2015), which are the most commonly collected item on beach cleans (Ocean Conservancy 2017). Chemicals that leach from smoked cigarettes include As, nicotine, PAHs, and metals (Moriwaki et al. 2009). Cigarette butt leachates can lead to toxic effects in multiple aquatic organisms (Micevska et al. 2006; Slaughter et al. 2011; Wright et al. 2015). Further, halogenated flame retardants are added to many plastic products that are not highlighted in the present study. Halogenated flame retardants have the second highest monetary value in the global market among plastic additives, following plasticizers (Clark 2014). Some halogenated flame retardants, such as hexabromocyclododecane
( $\mathrm{HCBD}$ ) and polybrominated diphenyl ethers (PBDEs) are known to be toxic, persistent, and bioaccumulative (Birnbaum and Staskal 2004) and are therefore listed under the Stockholm Convention as POPs (UNEP 2013). Jang et al. (2017) found high concentrations of HBCD in EPS debris found on beaches. They also demonstrated that EPS can be a source of HBCD to attached mussels (Jang et al. 2016). Thus, removing plastic debris from beaches or preventing such items from becoming marine debris not only prevents and reduces physical risks but also reduces risks relevant to chemical exposure.

\section{Estimates of PCB removal with microplastics removal}

Among the 4 locations, PCB concentrations found on individual microplastic samples ranged from 0.1 to $757 \mathrm{ng} / \mathrm{g}$ (Hirai et al. 2011; Rochman et al. 2014; IPW 2017; see Table 3 for all values). The largest range and highest concentration of PCBs was reported along the Hong Kong coastline $(10-757 \mathrm{ng} / \mathrm{g})$. The coast of Hawaii contained the largest amount of microplastic debris per area (maximum $1.57 \times 10^{3} \mathrm{~g} / \mathrm{m}^{2}$ ), with both gyres containing notably less microplastics in comparison to the 2 coastline sites. The $\mathrm{S}$ Atlantic gyre contained the least amount of microplastics with a maximum of $1.5 \times 10^{-6} \mathrm{~g} / \mathrm{m}^{2}$.

We estimated that the mass of PCBs that could be removed with microplastics within a $4500 \mathrm{~m} \times 1 \mathrm{~m}$ transect is much greater at the coasts than in the open ocean gyres (Figure 2, Map 1). Approximately 85000 times more PCBs could be removed from the Hong Kong coastline than from the same size transect in the N Pacific gyre, when maximum values were compared (Supplemental Data Appendix S15). Removal of PCBs also largely differed between regions. The mass of PCBs removed in the N Pacific gyre was more than 200 times greater than that removed in the S Atlantic gyre (maximum PCB removal: $2.0 \mathrm{ng}$ and $0.009 \mathrm{ng}$ per transect, respectively). Due to the high concentration of $\mathrm{PCBs}$ within plastic pellets along the Hong Kong coastline, the mass of PCBs that could be removed was approximately 12 times that removed in Hawaii (maximum PCB removal: $850 \mathrm{mg}$ vs $70 \mathrm{mg}$, respectively).

Although PCB concentrations within pellets found in Hawaii and the N Pacific gyre were similar (Table 3), total PCB removal was much higher in Hawaii. The important factor here was the amount of microplastics that could be collected, rather than the concentration of PCBs. The mass of microplastics that could be collected was much greater at the coastal locations of Hawaii (up to $7070 \mathrm{~kg}$ ) and Hong Kong (up to $1120 \mathrm{~kg}$ ), compared to the open ocean gyres ( $\mathrm{N}$ Pacific, up to $0.026 \mathrm{~g}$; S Atlantic, up to $0.0065 \mathrm{~g}$ ) (Figure 2, Map 2). Consequently, the coastal areas have the highest potential PCB removal values. As such, if plastic cleanup is to be used as a means to remove chemicals and legacy pollutants from the marine environment, these cleanup efforts should be aimed for locations with the greatest mass of plastic pollution per unit area rather than locations with the greatest concentration of chemical. 


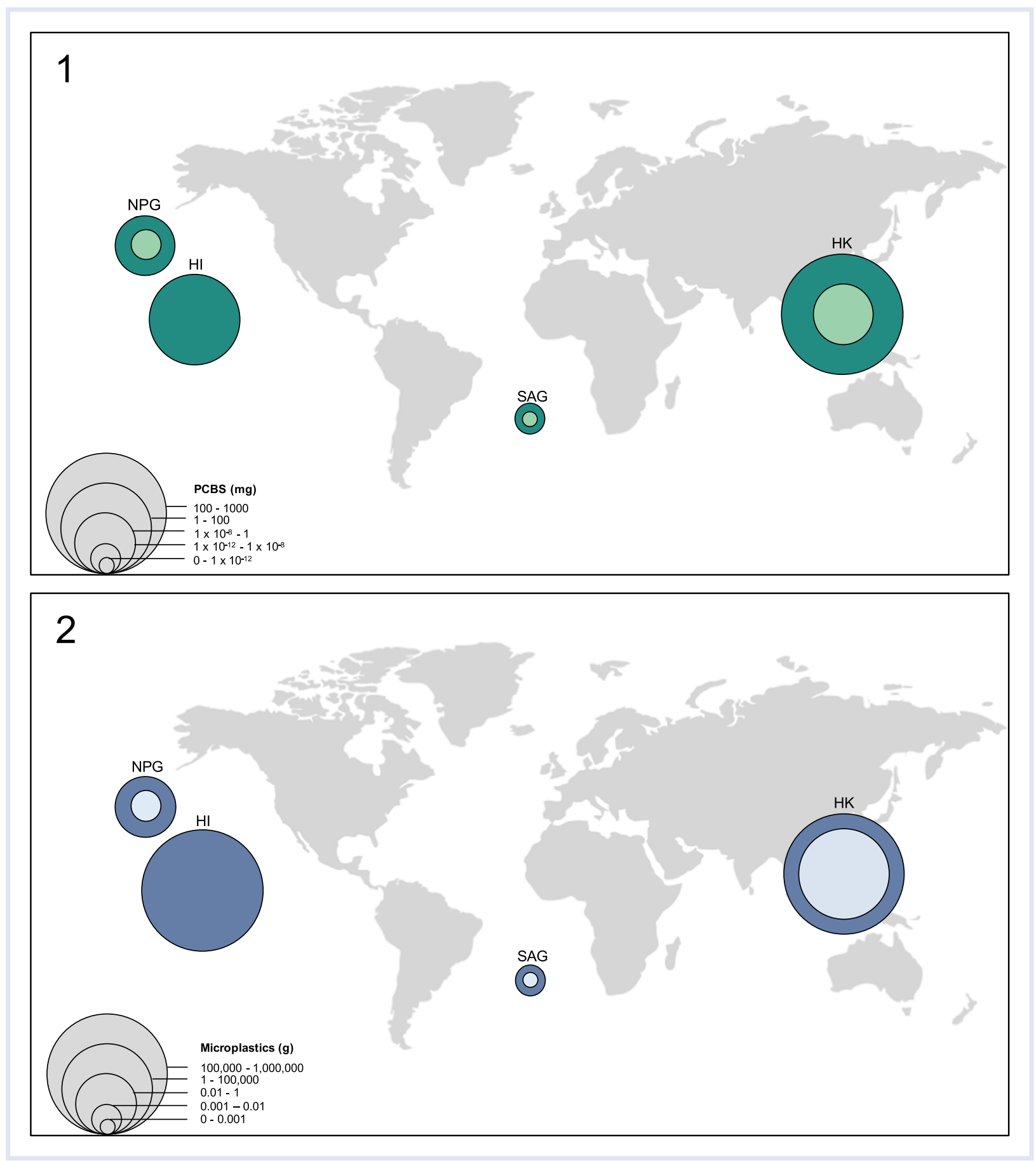

Figure 2. Mass of PCBs $(\mathrm{mg})$ estimated to be on microplastics collected from a $4500 \mathrm{~m} \times 1 \mathrm{~m}$ transect in each region (Hawaii, Hong Kong, North Pacific Gyre, South Atlantic Gyre). Light green indicates lowest estimates and dark green indicates highest estimates based on ranges of PCB concentrations (ng/g) and the total weight of microplastics in each area (1). Weight of microplastics (g) collected from a $4500 \mathrm{~m} \times 1 \mathrm{~m}$ transect at each location (Hawaii, Hong Kong, North Pacific Gyre, South Atlantic Gyre). Light blue indicates lowest estimates and dark blue indicates highest estimates for the range of values obtained. In Hawaii, there is no minimum value for both PCB removal and microplastics because the minimum amount of microplastics found in this location by Carson et al. (2011) was below the detection limit (2). $\mathrm{HI}=$ Hawaii; HK = Hong Kong; NPG = North Pacific gyre; SAG = South Atlantic gyre.

This finding is consistent with Sherman and van Sebille (2016), who calculated that optimal locations for microplastics removal are coastal areas. In these areas where greater amounts of plastic debris concentrate, they calculated that plastic collector technologies can remove $31 \%$ of total modeled microplastics mass by 2025 , compared to $17 \%$ in the N Pacific gyre. Plastic debris in open ocean gyres is also likely to be much older than coastal plastic debris, given that it will have travelled some distance to reach the open ocean, during which time it undergoes weathering, and thus leaching of chemical additives (Koelmans et al. 2016). At equilibrium, such particles will have relatively low concentrations of leachable chemicals (Hirai et al. 2011). Coastal areas contain relatively "young" particles with higher 
leachable content but also receive aged plastics from remote areas (Koelmans et al. 2016). Thus, by removing plastic debris from beaches, we prevent older plastic debris from reentering the ocean and new plastic items from entering the ocean and exposing marine life to their leachable chemical content.

Plastics are just one of multiple pathways for chemical exposure to marine life (Koelmans et al. 2016). When compared to other materials in the ocean, such as dissolved organic carbon and colloids, the fraction of organic chemicals sorbed by plastics is comparatively small (Koelmans et al. 2016). Still, due to their sorptive properties, plastics have been suggested as a management tool to "clean" chemicals from the water column (Zhu et al. 2011). Removing plastics that contain these chemicals from the marine environment removes these pollutants and their additive chemicals, reducing 1 exposure route to wildlife and decreasing potential harm. Thus, as a parks or resource manager, one can optimize effort by staying close to shore, that is, greater quantities of chemicals and microplastics can be removed by cleaning beaches, compared to the same size area of open ocean. Coastal removal is also more cost effective, requiring less time and resources to clean beaches and shorelines than to clean surface waters thousands of kilometers from land.

\section{CONCLUSION}

Plastic pollution prevention and cleanup is chemical pollution prevention and cleanup. Using a conservative data set, we show that prevention and cleanup efforts can reduce plastic additives and chemical contaminant loads in the ocean. We estimate that the total weight of 7 plastic items (bottles, bottle caps, EPS food and drink containers, cutlery, grocery bags, straws or stirrers, and food wrappers) that entered the ocean in 2015 was $87000 \mathrm{t}$ and that approximately $190 \mathrm{t}$ of 20 chemical additives entered the oceans with these plastic items. Prevention and cleanup of plastic debris can prevent additives from entering the ocean with plastics over time. We also demonstrate that shoreline cleanups can remove legacy pollutants, like PCBs, from the environment. We estimate that cleanup of plastic and associated chemicals is more efficient on shorelines, removing approximately 85000 times more PCBs than in oceanic gyres. Similar to other studies (Sherman and van Sebille 2016), our results demonstrate that cleanup nearer to the source (i.e., land) is more effective than open ocean cleanup. For PCBs, calculations show that their removal from beaches removes orders of magnitude more PCBs than does removal from the open ocean because much more microplastic can be collected per unit area. These results can be used to inform management actions, reiterating that coastlines and waterways should be prioritized for mitigation and cleanup strategies for both plastics (Sherman and van Sebille 2016) and chemicals.

Plastic debris is also a source of chemical additives to the marine environment. Upon leaching into the ocean, these chemicals will be diluted and thus may not be expected to cause impact. However, dilution cannot be the solution to pollution - with increasing quantities of plastics being added to the ocean, such concentrations will increase. Thus, although it is useful to talk about chemicals that accumulate on marine plastic debris, it is also critical to note the diverse and abundant chemical additives that plastics bring to the environment when they become debris, providing yet another reason for the prevention and removal of plastic debris from our oceans, waterways, and coastlines.

Acknowledgment-We would like to thank Greg Perkowski (Dart Container Corporation) and Jeff Wooster (Dow Chemical Company) for their input on our assumptions regarding the proportion of plastic types produced for certain products. We would also like to thank Professors Callum Roberts and Julie Hawkins (University of York) for their feedback on an earlier version of this study. Erik van Sebille was supported through funding from the European Research Council (ERC) under the European Union's Horizon 2020 research and innovation programme (grant agreement No 715386). Thanks to the Ocean Conservancy's International Coastal Cleanup 2014 volunteers for collecting and providing data on plastic debris items found along coastlines.

Disclaimer-The authors declare no conflicts of interest.

Data Accessibility - The data for this research concerning PCBs and microplastics are available online in the Supplemental Data. Much of the data on plastic additives was sourced directly from leading producers of plastics who are members of the American Chemistry Council (ACC) Plastics Division.

\section{SUPPLEMENTAL DATA}

Appendix S1. Average mass of each item of plastic debris, total number of items collected, and total mass calculations.

Appendix S2. Average mass of each item of nonplastic debris, total number of items collected, and total mass calculations.

Appendix S3. Estimated annual input of plastic to the ocean from waste generated by coastal populations worldwide in the year 2010, with projections for 2015, 2020, and 2025 in a business-as-usual scenario.

Appendix S4. Additive mass percentages provided by the ACC Plastics Division.

Appendix S5. Data sources for additive mass percentages for chemicals not included in ACC data.

Appendix S6. Mass of additives within each plastic debris item in the 2015 15\% scenario.

Appendix S7. Mass of additives within each plastic debris item in the 2015 40\% scenario.

Appendix S8. Mass of additives within each plastic debris item in the $202015 \%$ scenario.

Appendix S9. Mass of additives within each plastic debris item in the 2020 25\% scenario.

Appendix S10. Mass of additives within each plastic debris item in the 2020 40\% scenario.

Appendix S11. Mass of additives within each plastic debris item in the 2025 15\% scenario. 
Appendix S12. Mass of additives within each plastic debris item in the 2025 25\% scenario.

Appendix S13. Mass of additives within each plastic debris item in the 2025 40\% scenario.

Appendix S14. Results of aquatic toxicity tests for chemical additives found within the 7 common plastic items studied.

Appendix S15. Amount of PCBs that can be removed from a $4500 \mathrm{~m} 1 \mathrm{~m}$ transect in each region, calculated from the minimum and maximum PCB concentrations and minimum and maximum microplastics mass.

\section{REFERENCES}

Binetti R, Costamagna FM, Ceccarelli F, D'angiolini A, Fabri A, Riva G, Satalia S, Marcello I. 2008. The ISS National Inventory of Chemical Substances (INSC). Annali-Istituto Superiore Di Sanita 44(1):16-26.

Birnbaum LS, Staskal DF. 2004. Brominated flame retardants: Cause for concern? Environ Health Perspect 112(1):9-17.

Breivik K, Sweetman A, Pacyna JM, Jones KC. 2007. Towards a global historical emission inventory for selected PCB congeners - A mass balance approach 3. An update. Sci Total Environ 377:296-307.

Carson HS, Colbert SL, Kaylor MJ, McDermid KJ. 2011. Small plastic debris changes water movement and heat transfer through beach sediments. Mar Pollut Bull 62(8):1708-1713.

Carson HS, Lamso MR, Nakashima D, Toloumu D, Hafner J, Maximenko N, McDermid KJ. 2013. Tracking the sources and sinks of local marine debris in Hawai'i. Marine Environ Res 84:76-83.

Clark S. 2014. Plastic additives market by functions (property modifiers, property stabilizers, property extenders, processing aids), product types (plasticizers, flame retardants, impact modifiers, antioxidants, antimicrobials, UV stabilizers) and applications (plasticizers, flame retardants, impact modifiers, antioxidants, antimicrobials, UV stabilizers) - Global Opportunity Analysis and Industry Forecast, 2012 - 2020. Allied Market Research. [accessed 2017 Sep 9]. https://www.alliedmarketresearch.com/plastic-additives-market

Derraik JG. 2002. The pollution of the marine environment by plastic debris: A review. Mar Pollut Bull 44(9):842-852.

Engler RE. 2012. The complex interaction between marine debris and toxic chemicals in the ocean. Environ Sci Technol 46(22):12302-12315.

Eriksen M, Maximenko N, Thiel M, Cummins A, Lattin G, Wilson S, Hafner J, Zellers A, Rifman S. 2013. Plastic pollution in the South Pacific subtropical gyre. Mar Pollut Bull 68(1):71-76.

Fok L, Cheung PK. 2015. Hong Kong at the Pearl River Estuary: A hotspot of microplastic pollution. Mar Pollut Bull 99(1):112-118.

Gewert B, Plassmann MM, MacLeod M. 2015. Pathways for degradation of plastic polymers floating in the marine environment. Environ Sci: Processes Impacts 17(9):1513-1521.

Geyer R, Jambeck JR, Law KL. 2017. Production, use, and fate of all plastics ever made. Sci Adv 3(7):e1700782.

Gregory MR. 2009. Environmental implications of plastic debris in marine settings-Entanglement, ingestion, smothering, hangers-on, hitch-hiking and alien invasions. Philos Trans R Soc, B 364(1526):2013-2025.

Gregory MR, Andrady AL. 2003. Plastics in the marine environment. Plast Environ 379:389-390.

Hammer J, Kraak MH, Parsons JR. 2012. Plastics in the marine environment: The dark side of a modern gift. Rev Environ Contam Toxicol 220:1-44.

Hirai H, Takada H, Ogata Y, Yamashita R, Mizukawa K, Saha M, Kwan C, Moore C, Gray H, Laursen D, Zettler ER. 2011. Organic micropollutants in marine plastics debris from the open ocean and remote and urban beaches. Mar Pollut Bull 62(8):1683-1692.

Holmes LA, Turner A, Thompson RC. 2012. Adsorption of trace metals to plastic resin pellets in the marine environment. Environ Pollut 160:42-48.

[IPW] International Pellet Watch. 2017. PCBs: Global pollution map. [accessed 2017 Sep 9]. http://www.pelletwatch.org/maps/map-1.html

Jambeck JR, Geyer R, Wilcox C, Siegler TR, Perryman M, Andrady A, Narayan R, Law KL. 2015. Plastic waste inputs from land into the ocean. Science 347(6223):768-771.
Jang M, Shim WJ, Han GM, Rani M, Song YK, Hong SH. 2016. Styrofoam debris as a source of hazardous additives for marine organisms. Environ Sci Technol 50(10):4951-4960.

Jang M, Shim WJ, Han GM, Rani M, Song YK, Hong SH. 2017. Widespread detection of a brominated flame retardant, hexabromocyclododecane, in expanded polystyrene marine debris and microplastics from South Korea and the Asia-Pacific coastal region. Environ Pollut 231:785-794.

Jepson PD, Law RJ. 2016. Persistent pollutants, persistent threats. Science 352(6292):1388-1389.

Koelmans AA, Bakir A, Burton GA, Janssen CR. 2016. Microplastic as a vector for chemicals in the aquatic environment: critical review and modelsupported reinterpretation of empirical studies. Environ Sci Technol 50(7):3315-3326.

Lithner D, Larsson Å., Dave G. 2011. Environmental and health hazard ranking and assessment of plastic polymers based on chemical composition. Sci Total Environ 409(18):3309-3324.

Micevska T, Warne MSJ, Pablo F, Patra R. 2006. Variation in, and causes of, toxicity of cigarette butts to a cladoceran and microtox. Arch Environ Contam Toxicol 50(2):205-212.

Moriwaki H, Kitajima S, Katahira K. 2009. Waste on the roadside, 'poisute'waste: Its distribution and elution potential of pollutants into environment. Waste Manage 29(3):1192-1197.

Ocean Conservancy. 2017. International coastal clean-up reports. [accessed 2017 Sep 9]. http://www.coastalcleanupdata.org/reports

Ogata Y, Takada H, Mizukawa K, Hirai H, Iwasa S, Endo S, Mato Y, Saha M, Okuda K, Nakashima A et al. (2009. International pellet watch: Global monitoring of persistent organic pollutants (POPs) in coastal waters. 1. Initial phase data on PCBs, DDTs, and HCHs. Mar Pollut Bull 58(10): 1437-1446.

Rani M, Shim WJ, Han GM, Jang M, Song YK, Hong SH. 2014 Hexabromocyclododecane in polystyrene based consumer products: An evidence of unregulated use. Chemosphere 110:111-119.

Rios LM, Moore C, Jones PR. 2007. Persistent organic pollutants carried by synthetic polymers in the ocean environment. Mar Pollut Bull 54(8): 1230-1237.

Rochman CM, Lewison RL, Eriksen M, Allen H, Cook AM, Teh SJ. 2014. Polybrominated diphenyl ethers (PBDEs) in fish tissue may be an indicator of plastic contamination in marine habitats. Sci Total Environ 476: 622-633.

Sherman P, van Sebille E. 2016. Modeling marine surface microplastic transport to assess optimal removal locations. Environ Res Lett 11(1):014006.

Slaughter E, Gersberg RM, Watanabe K, Rudolph J, Stransky C, Novotny TE. 2011. Toxicity of cigarette butts, and their chemical components, to marine and freshwater fish. Tob Control 20(Suppl 1):i25-i29.

Suhrhoff TJ, Scholz-Böttcher BM. 2016. Qualitative impact of salinity, UV radiation and turbulence on leaching of organic plastic additives from four common plastics-A lab experiment. Mar Pollut Bull 102(1): 84-94.

Tawfik MS, BaAbdullah H. 2014. Migration levels of monostyrene in most vulnerable foods handled and stored in polystyrene containers and their impact on the daily intake. Pakistan J Food Sci 24(1):57-63.

Teuten EL, Saquing JM, Knappe DR, Barlaz MA, Jonsson S, Björn A, Rowland SJ, Thompson RC, Galloway TS, Yamashita R et al. (2009. Transport and release of chemicals from plastics to the environment and to wildlife. Philos Trans R Soc B 364(1526):2027-2045.

Thompson RC, Swan SH, Moore CJ, Vom Saal FS. 2009. Our plastic age. Philos Trans R Soc B 364(1526):1973-1976.

[UNECE] United Nations Economic Commission for Europe. 2013. Globally Harmonized System of Classification and Labelling of Chemicals (GHS). New York (NY) and Geneva (CH). [accessed 2017 Sep 15]. http://www. unece.org/fileadmin/DAM/trans/danger/publi/ghs/ghs_rev05/English/STSG-AC10-30-Rev5e.pdf

[UNEP] United Nations Environment Programme. 2013. Stockholm Convention on Persistent Organic Pollutants. Stockholm (SE). [accessed 2018 Jan 11]. http://www.austlii.edu.au/au/other/dfat/treaties/notinforce/2001/7.html

van Sebille E, Wilcox C, Lebreton L, Maximenko N, Hardesty BD, Van Franeker JA, Eriksen M, Siegel D, Galgani F, Law KL. 2015. A global 
inventory of small floating plastic debris. Environ Res Lett 10(12): 124006.

Westerhoff $P$, Prapaipong P, Shock E, Hillaireau A. 2008. Antimony leaching from polyethylene terephthalate (PET) plastic used for bottled drinking water. Water Res 42(3):551-556.

Wik A, Dave G. 2009. Occurrence and effects of tire wear particles in the environment-A critical review and an initial risk assessment. Environ Pollut 157(1):1-11.
Wilcox C, Mallos NJ, Leonard GH, Rodriguez A, Hardesty BD. 2016. Using expert elicitation to estimate the impacts of plastic pollution on marine wildlife. Mar Policy 65:107-114.

Wright SL, Rowe D, Reid MJ, Thomas KV, Galloway TS. 2015. Bioaccumulation and biological effects of cigarette litter in marine worms. Sci Rep 5:5.

Zhu H, Qiu S, Jiang W, Wu D, Zhang C. 2011. Evaluation of electrospun polyvinyl chloride/polystyrene fibers as sorbent materials for oil spill clean up. Environ Sci Technol 45(10):4527-4531. 